\title{
Prospective Validation of a Genomic Assay in Breast Cancer: The 70-gene MammaPrint Assay and the MINDACT Trial
}

\author{
William Audeh ${ }^{1}$, Lisa Blumencranz ${ }^{1}$, Heather Kling ${ }^{1}$, Harsha Trivedi $^{2}$, \\ Gordan Srkalovic ${ }^{2}$
}

${ }^{1}$ Agendia, Inc. Irvine, CA, ${ }^{2}$ Herbert-Herman Cancer Center, Lansing, MI

Correspondence: William.audeh@agendia.com Tel.: + 13108490561

Received: 15 October 2018 Accepted: 29 April 2019

Key words: Breast Cancer - Genomics * MammaPrint $\cdot$ MINDACT.

\begin{abstract}
MammaPrint was the first genomic assay in breast cancer to be validated with a prospective randomized trial, the MINDACT trial. The 70 gene MammaPrint assay was developed to determine the risk of distant metastasis in early stage breast cancer through gene expression analysis and was the first FDA cleared genomic assay for breast cancer. The assay identifies primary breast cancers likely to metastasize within the first five years of diagnosis and has clinical utility for helping to determine the expected benefit from adjuvant chemotherapy. The MINDACT Trial was the first trial of a genomic assay in breast cancer to provide prospective, randomized evidence of clinical utility for this important clinical question, identifying a significant proportion of patients who could safely forgo chemotherapy within a cohort of patients with high risk clinical characteristics. Nearly half of all patients (46\%) who would have been advised chemotherapy according to clinical guidelines were identified genomically by MammaPrint as being low risk and found to have equivalent rates of freedom from metastasis at 5 years with or without chemotherapy. Based upon the MINDACT trial, the ASCO Biomarker Guidelines now approve the use of MammaPrint to inform decisions regarding chemotherapy for women with clinically high-risk ER+ breast cancer, and as the only approved assay for use in women with 1-3 involved lymph nodes. Recent studies suggest information obtained from the 70-gene assay may also help inform decisions regarding endocrine therapy, as well as chemotherapy, targeted therapy and immunotherapy. Conclusion. The power of gene expression analysis in breast cancer, effectively illustrated with MammaPrint in the MINDACT trial, is now being explored through examination of the full transcriptome in breast cancer.
\end{abstract}

\section{Introduction - Bringing Genomic Anatomy to the Clinic}

The widespread availability and awareness of mammographic screening has led to the fortunate circumstance that most women diagnosed with breast cancer are diagnosed at a curable stage (1). The challenge for clinicians is therefore not only to achieve cure but do so with as little harm as possible for the individual with breast cancer. Given the well-known acute and long-term toxicities of chemotherapy, a fundamental requirement for precision oncology is to identify with certainty those patients who will benefit from chemotherapy and those who will not, in order to appropriately apply such therapy. The advent of rapid genomic tech- 
nology has allowed the development of clinically available genomic information, through gene expression microarray testing performed on cancer specimens. The 70-gene MammaPrint assay was the first such test to reveal the genomic "anatomy" of breast cancer, as it related to the most important aspect of early stage breast cancer, the likelihood of metastasis. The ability to detect this critical element of cancer biology carried clear implications for clinical utility when compared to classical morphologybased pathology (2). The ultimate proof of clinical utility for a genomic assay, however, requires a randomized prospective trial, and this has been achieved with the (Microarray in Lymph Node Negative and 1-3 Lymph Node Positive Disease May Avoid Chemotherapy) Trial MINDACT (3). This paper will review the basis for the MINDACT trial, the outcomes of the trial, and the implications it has provided for further application of genomic profiling in early breast cancer.

\section{MINDACT Trial Overview}

The MINDACT trial is the first prospective randomized trial reporting outcomes which illustrate the importance of genomic information for making appropriate treatment decisions in early stage breast cancer (3). The ability to routinely and rapidly analyze the genomic anatomy of breast cancers has revealed a level of information never before applied to a large adjuvant therapy trial, and the additional precision and clinical utility provided by gene expression analysis was clearly proven by the MINDACT trial (4). The primary finding from MINDACT, that a large proportion of women may be safely spared chemotherapy, avoiding its associated toxicities and costs without affecting their health outcomes, has broad implications for the quality of life of women with early stage breast cancer, as well as for health care costs
(5). The significance of the findings in the trial has continued to be appreciated since the publication in the New England Journal of Medicine in August 2016, with recognition of the practice-changing data by ASCO, NCCN, St Gallen, AJCC, and the health care insurance industry (6-9). The MINDACT trial has, however, also been considered complex in its design and extensive in the extent of data it generated, prompting the need for a broad and in-depth overview of the origins, rationale, and outcomes of this landmark trial.

\section{The Question To Be Answered by the MINDACT Trial}

The MINDACT Trial was designed to determine whether gene expression information from newly diagnosed early stage breast cancer (ESBC) could be used to identify breast cancers which were unlikely to benefit from chemotherapy and could safely avoid overtreatment and the associated toxicity (10). The importance of this question was emphasized by the fact that major clinical guidelines such as NCCN and St Gallen advised chemotherapy for a large proportion of ESBC to reduce the risk of metastatic recurrence based on clinical features and pathology $(8,9)$. This was particularly true for estrogen receptor positive (ER+) cancers, in which chemotherapy was added in addition to endocrine therapy based on clinical features alone, without clear evidence that chemotherapy was needed or beneficial in all cases. The toxicity and cost of chemotherapy mandated more concrete justification for its use, in the new era of genomic or "precision" oncology, where further information beyond simple immunohistochemical factors could be routinely obtained by assessing patterns of gene expression in primary breast cancers. 


\section{Genomic Anatomy Viewed Through the 70-Gene MammaPrint Assay}

The gene expression microarray utilized in MINDACT to provide this essential genomic information was the 70-gene panel known as MammaPrint, which had been developed and validated to predict the biological potential for metastasis in a primary breast cancer (2,11-13). The 70 genes which make up the MammaPrint genomic assay were discovered through an exhaustive, unbiased analysis of a cohort of breast cancers, collected and stored by the Netherlands Cancer Institute, from women who had undergone surgery, but had not received any systemic therapy for their cancers. Although the breast cancers from these women all appeared to be clinically and histologically similar, it was observed through long-term clinical follow-up that some women had remained entirely free of metastatic disease for many years, while others had experienced metastatic recurrences within the first five years after diagnosis (see Figure 1). Without the effect of systemic therapy to alter the outcome, this cohort provided a rare opportunity to identify the true biology of the potential for metastasis, a feature which could not be definitively identified by classical pathology alone. The genomic "anatomy", where the true pathology of cancer lies, was assessed with microarray technology, and gene expression from the entire genome was assessed for all cancers (2).

The gene expression pattern in cancers from women who did not have any incidence of metastatic recurrence within 5 years of diagnosis was compared to the gene expression pattern from cancers which had recurred with metastatic disease during that period, seeking differences which could distinguish the two groups. From this innovative and ground-breaking scientific study, 70 genes were identified, whose expression patterns could distinguish the non-metastasizing "Low Risk" breast cancers, (which may not have benefitted from adjuvant chemotherapy had it been given), from those early-metastasizing "High Risk" breast cancers, which clearly required systemic therapy. The 70 genes in the MammaPrint assay were found to be components of seven functional pathways involved in the metastatic process, providing the basis for their ability to predict the potential for metastasis (14, $15)$. The initial discovery was then validated in two other, larger cohorts $(12,13)$, and the consistent differences in clinical outcome were profound: an approximately $10 \%$ risk

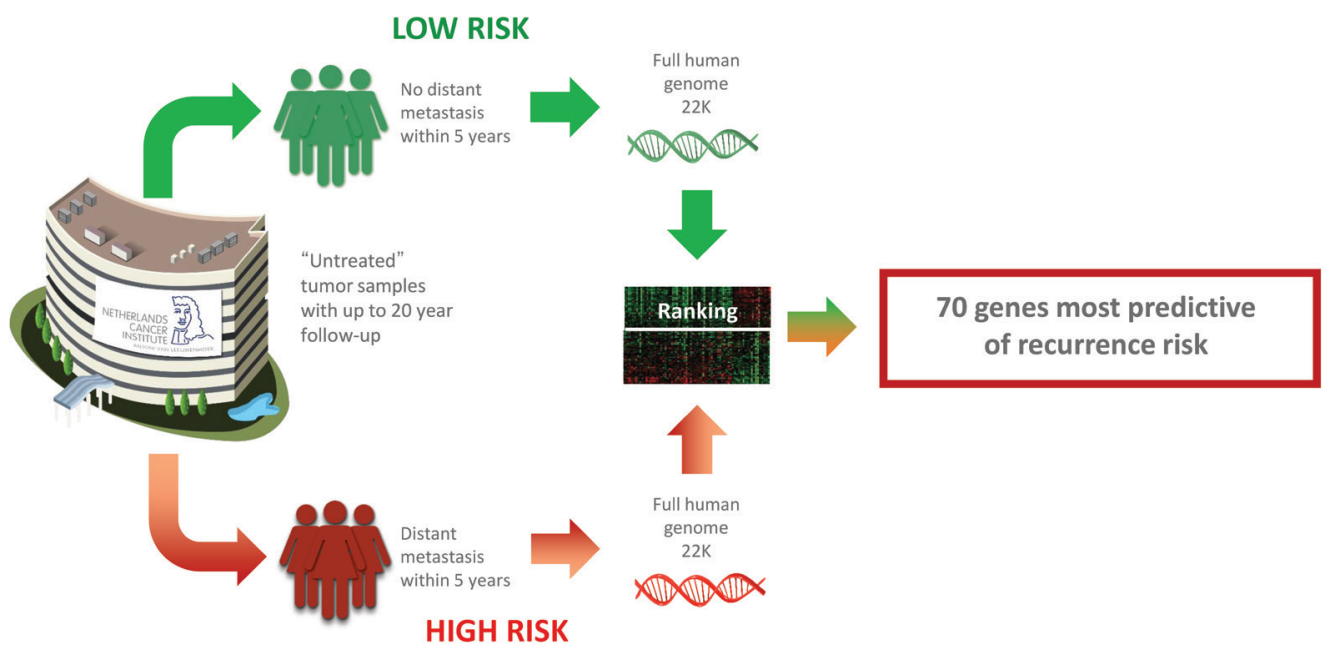

Figure 1. Development of the 70-gene MammaPrint signature. 
of metastasis at 10 years without any systemic therapy for cancers with a Low Risk 70 gene MammaPrint profile, and a nearly $30 \%$ risk of metastasis at 10 years with the High-Risk profile. With these data, MammaPrint became the first genomic assay in breast cancer to achieve FDA clearance (16). The 70 genes, in their respective patterns of under-expression or over-expression, effectively and accurately separated breast cancers into either of the two groups, without overlap; a breast cancer either had a significant likelihood of metastasis within the first five years, or it did not, as a binary quality.

The clear distinction between genomic High Risk and genomic Low Risk cancers, in the propensity to metastasize, paralleled the binary clinical decision-making process, in which a decision is made to administer chemotherapy or not, based on a clinically-derived assessment of metastatic risk. With the advent of this powerful genomic technology, readily accessible in the clinic, it was then necessary to ask which method of risk-assessment, clinical or genomic, was better able to predict the risk of metastasis, and therefore the need for systemic chemotherapy. This was the origin of the MINDACT trial.

\section{MINDACT Trial Design}

MINDACT was designed to determine if gene expression could identify individuals with genomic "Low Risk" breast cancers who were unlikely to benefit from chemotherapy and could safely avoid it. Importantly, MINDACT was not designed (or powered) to illustrate the extent of benefit of chemotherapy for genomically "High Risk" cancers, in part because cancers with a high risk of metastasis may or may not be chemo sensitive, may require additional targeted therapies in some cases, and may unfortunately relapse even with aggressive systemic therapy. The optimal treatment for such cancers continues to be the subject of intense research, although chemotherapy remains the standard of care at this time. In order to determine the answer to the MINDACT question, it was necessary to identify breast cancers which appeared to require chemotherapy based on clinical risk assessment ("clinically High Risk") but would not be predicted to benefit from chemotherapy by genomic, MammaPrint risk assessment (genomically Low Risk). If both methods of risk assessment were in agreement in all patients, it would then be clear that genomic assessment added nothing to standard clinical assessment, and no improvement could be made in the selection of patients requiring chemotherapy. However, if there were a substantial proportion of patients in which clinical and genomic risk assessment disagreed, it would be possible to determine the relative accuracy of both methods by randomizing such patients to have the chemotherapy decision based on either the clinical or genomic risk and compare the outcomes.

\section{Determining the Clinical Risk Assessment}

In order to make such a comparison, a standardized and reproducible method for clinical risk assessment was required. MINDACT was to be conducted in 9 European countries, with different languages and cultures. The solution to this was the use of a computer-based, universal algorithm for clinical risk assessment, Adjuvant!Online (17). This program, a compendium of clinical data from numerous large prospective adjuvant therapy trials in breast cancer had been available and familiar to all clinicians for many years and provided an estimate of Overall Survival without chemotherapy at 10 years. (For the MINDACT trial, a modified version which also integrated HER2 was employed).

The question also arose as to what level of "clinical risk" warranted the use of chemotherapy. In addition to the diversity of 
language and culture across the MINDACT study sites, there were different regional thresholds for the level of risk which justified the administration of chemotherapy, as well as individual physician opinions regarding when chemotherapy was warranted. The common thread amongst all breast cancer clinicians, however, was acknowledging the importance of integrating the patient's own preferences when making the important chemotherapy decision $(18,19)$. The risks and toxicity of chemotherapy were of course of great concern to patients; temporary risks such as hair loss, fatigue, nausea; more permanent risks such as neuropathy and cognitive dysfunction, as well as the rare lifethreating risks of acute leukemia and cardiac disease. The patient-based threshold for enduring chemotherapy to reduce the risk of breast cancer recurrence versus the risk of toxicity of chemotherapy was therefore assessed through polling women regarding their opinions. Prior studies from the United States, Australia and Europe had documented wide variation in patient thresholds for the necessary magnitude of chemotherapy benefit, ranging from $0.5 \%$ to over $5 \%$ (18-21). The result obtained from the women polled for the design of the MINDACT trial was that chemotherapy would be worth the toxicity for most women with ESBC if it provided a greater than $2 \%$ benefit for breast cancer specific survival (BCSS). This threshold then required calculation of the absolute level of risk which is improved by at least $2 \%$ with the use of chemotherapy, based on prior clinical studies. The generally-recognized benefit of chemotherapy, as reported by the Early Breast Cancer Trialist's Collaborative Group (EBCTCG) in the so-called Oxford Overview, is to improve survival by approximately $25 \%$, based on data from three decades of adjuvant trials in breast cancer (22, 23). A $2 \%$ absolute benefit of chemotherapy, would be obtained when the overall risk is $8 \%$, as this would constitute a relative benefit of $25 \%$. With $8 \%$ risk being the minimum risk for which chemotherapy is justified, any patients with a clinical estimate of Overall Survival, with endocrine therapy but without chemotherapy, of $92 \%$ or higher at 10 years by Adjuvant!Online would be categorized as Clinical Low Risk, and not likely to derive meaningful benefit from adjuvant chemotherapy. The clinical characteristics of the Clinical Low Risk group included T1a and $\mathrm{T} 1 \mathrm{~b}$ tumors of any grade, T1c tumors of Grade 1 or 2, and Grade 1 tumors of $3 \mathrm{~cm}$ or less. All others with an expected benefit of chemotherapy of greater than $2 \%$ would be classified as Clinical High Risk, with the potential benefit sufficient to advise chemotherapy (10).

\section{MINDACT Trial Methods}

How often were clinical Risk (according to Adjuvant! Online and genomic risk, (according to MammaPrint risk assessment) in agreement, and when they disagreed, which method of risk assessment was better able to predict the need for chemotherapy? This central question for the MINDACT trial were answered by conducting both clinical and genomic risk assessment on every patient enrolled in the trial. If there was agreement, or concordance, between the clinical and MammaPrint methods for identifying a Low Risk patient (clinically Low/genomically Low, or $\mathrm{cL} / \mathrm{gL}$ ), then no chemotherapy would be advised, while if both agreed in identifying a High-Risk patient (clinically High/genomically High, or $\mathrm{cH} / \mathrm{gH}$ ), then chemotherapy would be routinely advised, and no randomization would be required. However, for those patients classified as clinically High Risk by Adjuvant!Online but were identified as genomically Low Risk by MammaPrint ( $\mathrm{cH} / \mathrm{gL})$, i.e. discordant, these patients would be randomly assigned to have the chemotherapy decision based on clinical risk or genomic risk. For those 
patients whose treatment decision would be based on genomic Low Risk with MammaPrint, and no chemotherapy given, their rates of recurrence with distant metastasis needed to be at least as low as those patients whose treatment was determined by their clinical High-Risk category and did receive chemotherapy. Therefore, MINDACT was a "non-inferiority" trial, designed to determine whether the outcome of the treatment decision based on genomically-assessed risk would be as good as, or not inferior to, the outcome when the therapy decision was based on clinical risk assessment. In terms of statistical significance, the non-inferiority goal would be a lack of statistically significant difference between the clinical outcomes of the two groups.

\section{The Relevant Clinical Endpoint in MINDACT: Distant Metastasis}

Because the purpose of chemotherapy is to reduce metastatic recurrence, the main life-threatening aspect of breast cancer, the optimal endpoint for making the comparison between the groups in MINDACT is the incidence of distant metastasis (24). Many clinical endpoints are collected in clinical trials, but are less relevant to the question of whether chemotherapy is needed to reduce the rate of distant metastatic recurrence: Disease Free Survival or DFS includes events such as second primary cancers and local in-breast recurrence, clinical events which, while undesirable, are not the primary reason chemotherapy is given, and Overall Survival, or OS, includes deaths from any cause, and does not distinguish those who have experienced metastatic recurrence from those who have not. As described in a consensus statement in the Journal of Clinical Oncology regarding relevant endpoints:

"The separation of distant as a specific end point is also very important for ancillary studies involving microarray analysis and for developing genetic panels for use in determining prognosis and/or response to treatment. In these situations, distant disease recurrence is often used as a marker for survival to increase statistical power because there is such a strong correlation between these end points and because there will be more distant events than deaths. Using a combined regional/distant end point would dilute the correlation with survival and weaken the discriminatory power of the analysis" (24).

The optimal endpoint, therefore, in a study to validate the clinical utility of a genomic assay, is one which registers distant metastasis or death ("distant metastasis free survival" or DMFS). If distant metastases and only deaths due to breast cancer are registered, the endpoint is "distant metastasis free interval", or DMFI. The MINDACT trial was therefore designed and powered to determine whether DMFS for the clinically High Risk/MammaPrint Low Risk group would be the same at five years for those who received chemotherapy and those who did not. The assumption was made by the MINDACT investigators that the minimum acceptable outcome for DMFS for the cohort not receiving chemotherapy was required to be at least $92 \%$ at 5 years, and if it appeared that the rate of metastatic recurrence during the conduct of the trial appeared to exceed this rate, the trial could be stopped early, for patient safety.

\section{The Role of Chemotherapy in Reducing Metastatic Recurrence of Breast Cancer}

The Early Breast Cancer Trialist's Collaborative Group (EBCTCG) conducts periodic meta-analyses of the long-term outcomes of three decades of adjuvant therapy trials in breast cancer, the results of which have formed the basis of the standard of care and clinical treatment guidelines for many years 
$(22,23)$. Such meta-analyses provided proof of the overall benefit chemotherapy in reducing metastatic recurrence and improving overall survival, although only for a small proportion of women with breast cancer, as well as the incremental benefit of the addition of anthracyclines and taxanes to chemotherapy regimens. A major observation obtained from over 15 years of follow-up of tens of thousands of women with ESBC, is that the effect of chemotherapy in reducing metastatic recurrence is seen primarily during the first five years after diagnosis. The five years of follow-up reported in the MINDACT trial were therefore considered sufficient to identify all patients benefitting from chemotherapy. Although metastatic recurrences continue to occur after five years, almost exclusively in estrogen receptor positive breast cancer, such "late" recurrences occur at the same rate in women who received chemotherapy as in those who did not, indicating the need for interventions other than chemotherapy to reduce these recurrences, such as extended endocrine therapy or targeted agents.

\section{MINDACT Trial Enrollment}

From 2007 through 2011, 6,693 patients with breast cancer were enrolled, across 9 European countries, in over 110 individual sites. Enrolled patients were required to have a pathology-confirmed diagnosis of breast cancer, a tumor stage of T1, T2 or operable T3, and from 0 to 3 positive lymph nodes. The majority of enrolled patients had ER+ breast cancer (88.4\%), and ranged in age from 23 to 71 , with the median age being 55. Importantly, one third (33.2\%) of MINDACT patients were less than 50 years of age.

\section{Main Results of the MINDACT Trial}

Figure 2 shows the distribution of patients classified according to clinical risk by Adjuvant! Online and genomic risk by MammaPrint. Approximately half of all patients were classified as clinically Low Risk, and half as Clinically High Risk, while MammaPrint identified $64 \%$ as genomically Low Risk, and $36 \%$ as genomically High Risk. The comparison of clinical risk assessment with genomic risk assessment revealed agreement, or concordance, in two thirds (68\%) of all patients, with $41 \%$ clinically and genomically Low Risk (cl/gL) and 27\% clinically and genomically High Risk (cH/ $\mathrm{gH}$ ), supporting the continued importance of clinical factors in estimating the risk of metastatic recurrence. However, clinical risk was primarily concordant with genomic risk in identifying Low Risk patients, who were

$(\mathrm{N}=6693)$

Clinical Risk = Adjuvant!Online

Genomic Risk = 70-gene MammaPrint signature
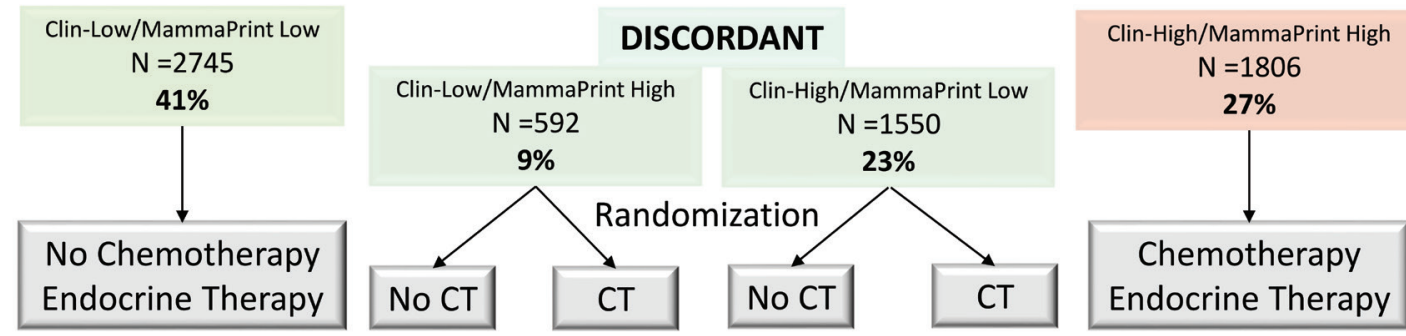

Figure 2. Distribution of Clinical Risk and Genomic Risk in the MINDACT Trial. From Ref. 3 Cardoso (2016). 
unlikely to benefit from chemotherapy, with 4 out of 5 being concordant, and in only 1 one out of 5 did genomic analysis provide additional information. In contrast, for the $50 \%$ of MINDACT patients identified to be clinically High Risk, in only 1 of 2 was there concordance with the genomic risk, with an equal proportion having a discordant, genomic Low Risk. This finding supports the original rationale of the MINDACT trial, to seek to identify, by gene expression patterns, those patients who may be potentially overtreated when the decision to administer chemotherapy is based on clinical risk assessment alone.

\section{The Primary Test Group in MINDACT: Clinically High Risk but MammaPrint Low Risk Patients}

1550 patients were in the discordant $\mathrm{cH} /$ gL group, with half randomly assigned to receive chemotherapy based on the clinical High-Risk assessment, or not to receive chemotherapy, based on their genomic Low Risk assessment. Figure $3 \mathrm{a}$ shows the outcome for the $\mathrm{cH} / \mathrm{gL}$ group which did not receive chemotherapy (with 100\% compliance "Per
Protocol" with this treatment decision). The DMFS at 5 years for this group was $94.7 \%$, with a confidence interval ranging from $92.5 \%$ to $96.2 \%$, well above the threshold of $92 \%$ set by the MINDACT investigators. The MINDACT trial therefore met its required endpoint and is considered a positive trial.

Most important, however, was the comparison of the DMFS at 5 years with the $\mathrm{cH} /$ gL cohort who did receive chemotherapy. Shown in Figure $3 \mathrm{~b}$ is this comparison, using the "Intent to Treat" cohorts, taking into account the small numbers of patients (approximately $12.7 \%$ ) in both groups who did not follow their assigned treatment. The reasons for not following the treatment randomly assigned by the protocol were described in the Supplementary Section of the NEJM publication (3), and were primarily due to patient preference; some assigned to chemotherapy declined it, and some assigned to no chemotherapy requested to be treated. The DMFS for the group assigned to receive chemotherapy was $95.9 \%$, while the group assigned to no chemotherapy was $94.4 \%$, a numerical difference of $1.5 \%$ which was not statistically significant, with a p-value of 0.267 . The MINDACT trial

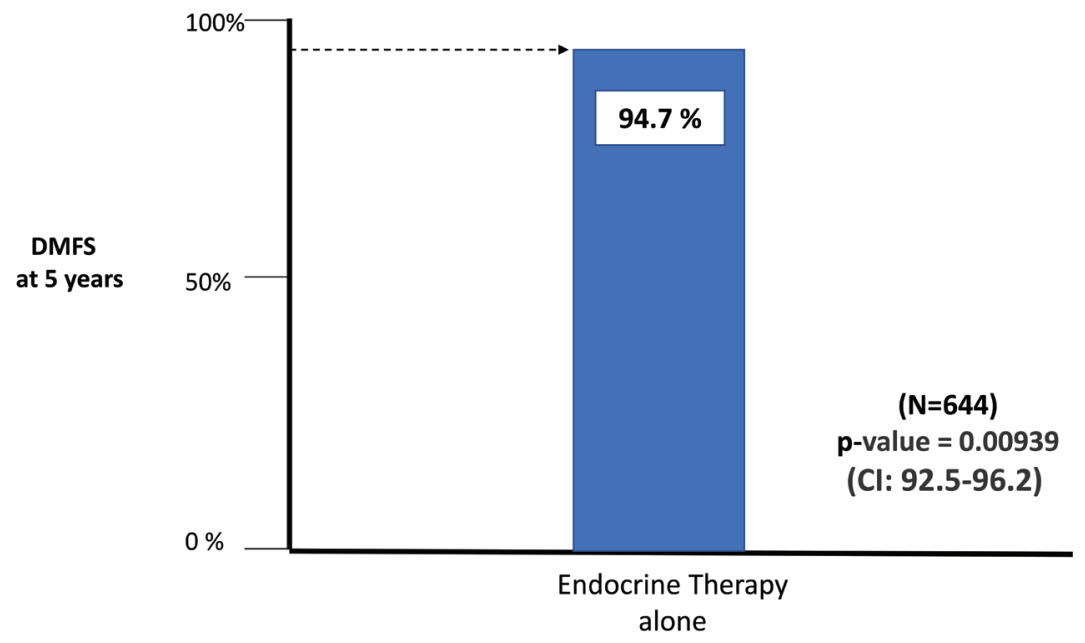

Figure 3a: Clinical High Risk/MammaPrint Low Risk Treated Without Chemotherapy (Per Protocol; (Distant Metastasis Free Survival). From ref. 3 Cardoso (2016). 


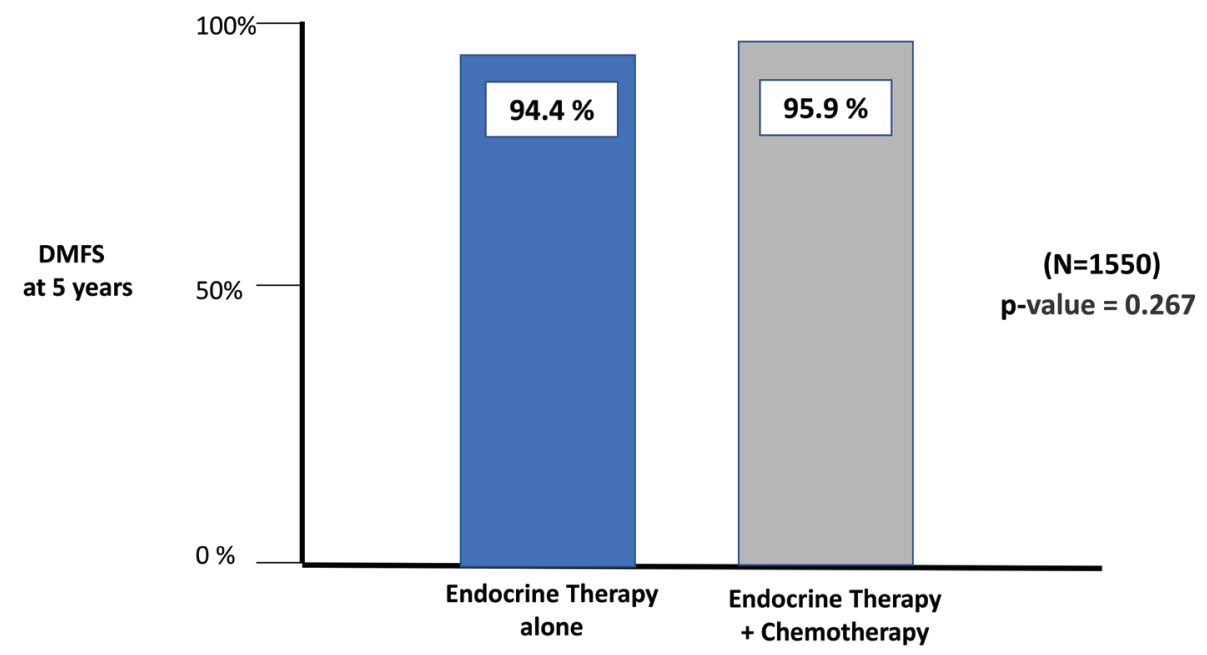

Figure 3b. Clinical High Risk/MammaPrint Low Risk treated with/without chemotherapy (intent to treat; (Distant Metastasis Free Survival). From ref. 3 Cardoso (2016).

had sufficient patients enrolled, and recurrence events observed, to detect a clinically meaningful difference, if it existed, between the chemotherapy and no chemotherapy groups. The lack of a statistically significant difference in the frequency of metastatic recurrence between these groups supported the hypothesis that MammaPrint Low Risk patients could safely avoid chemotherapy, even when clinical High-Risk factors were present.

\section{The "Non-Significant" $1.5 \%$}

In the Editorial by Drs Hudis and Dickler which accompanied the MINDACT trial publication in the NEJM, the issue of the $1.5 \%$ numerical difference was addressed (4). In their words, "a difference of $1.5 \%$, if real, might mean more to one patient than to another". Acknowledging that the threshold for accepting the toxicity of chemotherapy in return for some degree of protection from recurrence is a matter of individual preference, they agree that MammaPrint can identify patients "in whom any plausible benefit of chemotherapy would be modest". Drs Hudis and Dickler concluded that "On the basis of the MINDACT study, clinicians may consider ordering the 70-gene signature for patients in line for chemotherapy who hope to forgo it on the basis of a possibly low genomic risk."

\section{The Lymph Node Positive Patients in MINDACT}

Nearly 1400 patients with 1-3 involved lymph nodes were enrolled in MINDACT, the largest cohort of node positive patients reported in a randomized controlled trial involving genomic profiling of breast cancer. Node positive patients represented 21\% of the entire enrolled population. However, within the important primary test group of clinically High Risk/MammaPrint low risk patients, $48 \%$, or 709 were node positive. Figure $3 \mathrm{c}$ shows the DMFS rates at 5 years for the 1-3 Lymph Node positive patients, with those receiving chemotherapy at $96.3 \%$ and those not receiving chemotherapy at 95.6\% DMFS. As with the $\mathrm{cH} / \mathrm{gL}$ group as a whole, the p-value of 0.724 indicated no statistically significant benefit to the addition of chemotherapy for this large cohort with lymph node positive breast cancer. 


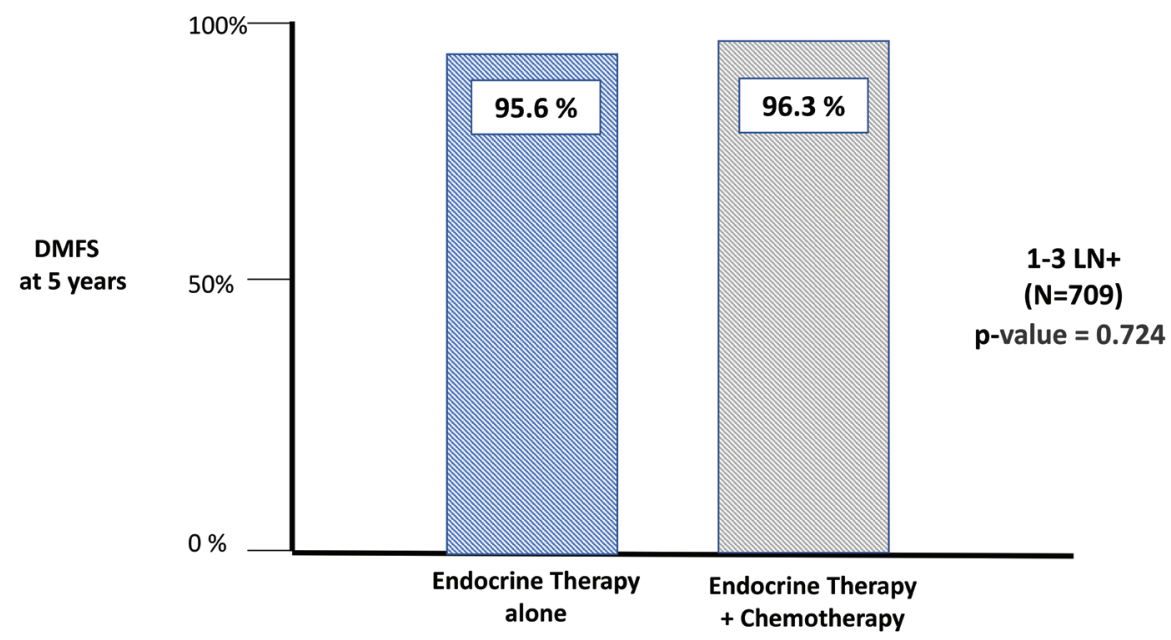

Figure 3c. Clinical High Risk/MammaPrint Low Risk treated with/without chemotherapy (intent to treat) 1-3 LN+. From ref. 3 Cardoso (2016).

The implications of this critical finding are that cancers with the biological capacity to reach regional lymph nodes do not always have the capacity for distant metastases, and my not require chemotherapy, in contrast to recommendations in most clinical guidelines. MINDACT provides the first and only prospective, randomized data in over 700 patients with 1-3 lymph node positive breast cancer in which genomic profiling can identify those patients who can safely avoid chemotherapy.

\section{The Effect of Genomic Risk}

\section{Assessment in Early Stage Breast}

\section{Cancer}

Additional endpoints beyond the primary goal of identifying clinically high-risk patients who could safely avoid chemotherapy were also analyzed. The effect of determining the need for chemotherapy for all patients based either on clinical risk assessment or genomic risk assessment as measured by DMFS was assessed. For those MINDACT patients in whom clinical risk assessment was used to determine the need for chemotherapy, the DMFS at 5 years was
95\%, while those patients treated according to their MammaPrint risk assessment had a 5 -year DMFS of $94.7 \%$. There was no statistically significant difference in these clinical outcomes; however, the MammaPrint risk assessment allowed $46 \%$ of clinically highrisk women to safely avoid chemotherapy. The benefits of avoiding unnecessary, toxic chemotherapy, for quality of life are likely to be substantial, as well as the health economic benefits of avoiding costly therapy and managing its side effects. Cost-effectiveness data from MINDACT are being analyzed, although previous studies have already documented the cost-effectiveness of the 70 gene MammaPrint assay $(5,25)$.

\section{Randomization of Chemotherapy Regimen}

Patients receiving chemotherapy in either the concordant High-Risk cohort or the discordant cohorts randomized to receive chemotherapy were offered an optional secondary randomization to either standard of care anthracycline-containing regimens or the non-anthracycline study regimen, docetaxel/capecitabine. Of the 2877 pa- 
tients receiving chemotherapy, 1806 (63\%) were in the clinical High/ Genomic HighRisk group, 775 (27\%) were Clin High/Genomic Low, and 292 (10\%) were Clin Low/ Genomic High Risk. Not all patients participated in the secondary randomization, with 2227 (77\%) receiving standard of care regimens, and 650 (23\%) treated with docetaxel/ capecitabine. The choice of regimen did not affect clinical outcomes, which were similar in the standard and non-standard arms (26).

\section{Questions Arising from MINDACT: Is MammaPrint Predictive of Chemotherapy Benefit?}

MINDACT was designed and patient enrollment numbers calculated to answer the question of whether MammaPrint could identify women with genomically Low Risk ESBC who could safely avoid chemotherapy. It was not designed to answer whether chemotherapy would benefit women with genomically High Risk breast ESBC. The design of MINDACT was such that all patients with discordance between clinical and genomic risk assessment were randomized to have the decision to administer chemo- therapy based on one of the two methods. Although the largest discordant group was the primary test group described above, Clinically High Risk and Genomically Low Risk, ( $n=1550,23 \%$ of total), there was also a small cohort in which the Clinical Risk was Low and Genomic Risk High ( $\mathrm{n}=592$, 9\% of total). The clinical characteristics of this group were low risk due primarily to small tumor size $(98 \% \mathrm{~T} 1)$ and low to intermediate grade $(85 \%)$. Although the majority of patients in this cohort were estrogen receptor positive, approximately $12 \%$ were also HER2+, while 9\% were classified clinically as "triple negative".

This discordant group also underwent randomization, per the protocol design, with the group receiving chemotherapy based on genomic High Risk having a 98.1\% DMFI at 5 years, versus DMFI of $95.6 \%$ for the group not receiving chemotherapy due to Low Clinical Risk, a numerical difference of 2.5\% which did not reach statistical significance ( $p$ value 0.282 ) (Figure 4). Due to the small benefit of chemotherapy predicted by the clinical Low Risk classification, a significantly larger number of randomized patients, at least 2000, would have been required to de-

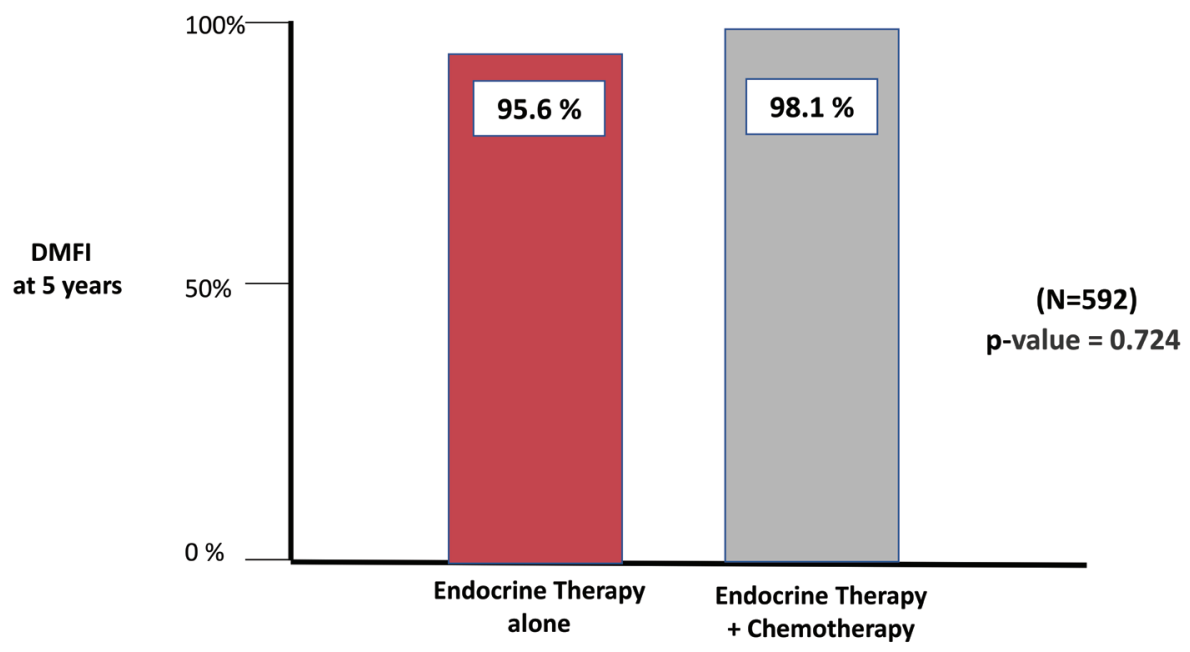

Figure 4. Clinical Low Risk/MammaPrint High Risk treated with/without chemotherapy (intent to treat) Distant Metastasis Free Interval (DMFI). From ref. 3 Cardoso (2016). 
tect a statistically significant benefit of chemotherapy in the MammaPrint High Risk cohort (27). As the MINDACT trial was not powered or designed to determine the benefit of chemotherapy in MammaPrint High Risk patients, MINDACT does not provide definitive data to rule in or rule out the benefit of chemotherapy in MammaPrint High Risk patients. However, the lack of statistical significance for the benefit of chemotherapy in clinically low risk patients with a High Risk MammaPrint index does not affect the predictive value of the assay.

\section{When Is a Test "Predictive"?}

Diagnostic tests are generally used to predict the likelihood of a medical condition and have both a positive predictive value (PPV) and a negative predictive value (NPV) for predicting the presence of the condition. Diagnostic tests may also be used to predict the likelihood of responding or not responding to a specific therapy. A PPV in this context is defined as the proportion of patients with a "positive" test result who will benefit from a therapy, while the NPV is the proportion of patients with a "negative" test result who will not benefit from the therapy. Most diagnostic tests have significantly different predictive value if the test is "negative" or "positive".

In breast cancer, the use of molecular tests for the presence of estrogen receptor protein (ER) and amplification of the HER2 gene are considered standard of care in predicting response to anti-estrogen therapy and HER2-targeted therapy, respectively. Yet the NPV and PPV of these tests vary significantly. The presence of ER predicted a $60 \%$ rate of response in ER+ metastatic breast cancer (28), while when ER was absent, the rate of response was $5-8 \%$. Therefore, the PPV of the ER test was $60 \%$, while the NPV, the ability to identify non-responders, was far greater, at $92-95 \%$. For the prediction of response to endocrine therapy, the NPV of ER is of greater utility and accuracy.

With detection of amplification of HER2 as a predictive test for the likelihood of response to HER2-targeted therapy, the original studies of single agent trastuzumab in metastatic breast cancer observed a 35\% response rate in HER2-amplified (FISH+) patients, and a $7 \%$ response rate in patients without HER2 amplification (29). Therefore, the PPV of the presence of amplification of HER2 for predicting response to trastuzumab was $35 \%$. The NPV of the absence of HER2 amplification was far greater, at 93\% in this trial, and approaching $99 \%$ in recent trials (30), predicting lack of benefit of trastuzumab.

\section{The Predictive Value of MammaPrint}

In this context, the question of predictive value may also be applied to MammaPrint. MammaPrint is indeed predictive. The MINDACT trial tested the NPV of MammaPrint for the potential benefit from chemotherapy in preventing distant metastasis. In other words, would a Low Risk MammaPrint index predict the absence of benefit from chemotherapy? The answer from the MINDACT trial was "yes", in that the administration of chemotherapy to patients with a MammaPrint Low Risk index did not yield a statistically significant difference in freedom from metastasis, although a numerical difference of $1.5 \%$ in DMFS was reported. Therefore, the NPV of MammaPrint for chemotherapy benefit is $98.5 \%$, in that MP correctly identified $98.5 \%$ of ER+, clinically High-Risk patients who would not derive benefit from chemotherapy. The NPV of MammaPrint for predicting absence of chemotherapy benefit is equal to the NPV of ER or HER2 in predicting the absence of benefit to tamoxifen or trastuzumab, respectively.

Does a High-Risk MP score predict the presence of benefit from chemotherapy? In 
other words, what is the PPV of MammaPrint? This question cannot be answered by the MINDACT trial, as this would have required that all enrolled patients with a HighRisk MP score be randomized to receive either chemotherapy or no chemotherapy, even those with concordant clinical high risk, a design which would have been unethical by $21^{\text {st }}$ century clinical trial standards. The clinically Low Risk cohort (greater than $92 \%$ overall survival at 10 years without chemotherapy, according to clinical/pathologic features) within the MammaPrint High Risk group were randomized, but with such a good clinical prognosis, the ability to detect a further benefit was exceedingly small. Of these clinically Low Risk patients, 95.6\% were free of distant metastases (DMFI) with endocrine therapy alone after 5 years, while the addition of chemotherapy increased the proportion without metastasis to $98.1 \%$, a difference of $2.5 \%$. Since the remaining risk of metastasis, despite endocrine therapy, was $4.4 \%$, the MP High Risk patients benefitted from chemotherapy by $2.5 \%$ of the $4.4 \%$, a relative risk reduction of $56 \%$ of the residual risk. A very similar magnitude of relative risk reduction has been observed in other, non-randomized MammaPrint cohorts, in which a pooled series of $541 \mathrm{ER}+$ patients with both clinical high and low risk treated with chemotherapy and endocrine therapy had a distant disease free survival (DDFS) of $88 \%$, in comparison to $76 \%$ DDFS for those receiving only endocrine therapy (31), an absolute reduction of $12 \%$, and a relative risk reduction of $50 \%$, in keeping with the MINDACT data.

\section{ASCO Clinical Practice Guidelines, NCCN Guidelines, and the MINDACT Trial}

The results of the MINDACT trial were reviewed by the American Society of Clinical Oncology (ASCO) Clinical Practice Guide- line Expert Panel in an update in 2017 devoted entirely to the trial (6)). Based on these data, the updated guidelines stated that MammaPrint may be used in clinically highrisk ER+, HER2-, Lymph Node negative and 1-3 Lymph node positive breast cancer to "inform decisions in withholding adjuvant systemic chemotherapy". For women with 1-3 positive lymph nodes, the guidelines further specified that "such patients should be informed that a benefit of chemotherapy cannot be excluded, particularly in patients with greater than one involved lymph node." However, the guideline recommended that other genomic assays should not be used in lymph node positive patients. MammaPrint was identified as the first and only genomic assay which could be used in this group.

In 2018, over two years after the publication of the MINDACT trial data, the NCCN updated their Breast Cancer Guideline to acknowledge that with the MINDACT trial, MammaPrint was the only risk of recurrence genomic assay with Level 1 evidence in both lymph node negative and lymph node positive breast cancer (32). The only other risk of recurrence genomic assay recognized to also have Level I evidence limited only to lymph node negative breast cancer, was the 21-gene Oncotype Dx assay, based upon the Trial Assigning Individualized Options for Treatment (TAILORx) (33).

\section{Prospective Randomized Trials of Genomic Assays in Breast Cancer: MINDACT and TAILORx}

MINDACT was the first reported prospective randomized trial of a risk of recurrence genomic assay in breast cancer. Two years after the publication of the MINDACT trial, the second, and largest such trial of this kind, (with over 10,000 patients enrolled), TAILORx which evaluated the 21-gene OncotypeDx assay, also reported the prospective and randomized arms of the trial (33). 
Unlike MINDACT, in which the primary goal was to confirm the safety of withholding chemotherapy in clinically high risk/ MammaPrint low risk patients, TAILORx was instead intended to clarify whether a specific risk of recurrence "score" (RS) could identify patients who could safely avoid chemotherapy. Unlike MINDACT, TAILORx was limited to lymph node negative patients. Clinical risk category, as had been determined by the MINDACT trial, was assessed in TAILORx, but was not used in the randomization or patient selection for enrollment. The randomized cohort was not balanced for clinical risk, in that $74 \%$ of the randomized patients were clinical "Low Risk" as defined in the MINDACT trial. The cohorts randomized to receive chemotherapy followed by endocrine or endocrine therapy alone, were limited to those with RS 1125 , with no randomization for RS $0-10$, or RS 26 and above. The results, without subset analysis, showed no benefit in DFS with the addition of chemotherapy with RS 11-25. However, subset analysis revealed that these findings did not apply equally to all ER+, lymph node negative women, with a chemotherapy benefit of $5.8 \%$ seen for women 50 years old and younger with RS 16-25. The low event rate in this study led some (34) to question whether the predominance of clinical low risk patients precludes drawing any definitive conclusions regarding the utility of an intermediate RS for determining the need for chemotherapy with this assay.

\section{Future Directions for MammaPrint and Gene Expression Profiling in Breast Cancer}

The MINDACT trial proved the clinical utility of stratifying breast cancers as having a Low or High Risk MammaPrint Index, through the expression patterns of 70 genes, for identifying patients who could safely forgo chemotherapy. Within the Low and High-
Risk categories, further stratification of the range of the MammaPrint index has provided additional information with clinical utility which could not be obtained through clinical features or pathology. Within the Low Risk MammaPrint Range, from $>0.00$ to +1.00 , all patients may safely forgo chemotherapy, and ER+ patients are routinely treated with 5 years of endocrine therapy alone. However, a subset of these MammaPrint Low Risk patients, with indices from $>0.355$ to +1.00 appear to have an extremely low risk of recurrence, with or without 5 years of endocrine therapy, for over 20 years from diagnosis (35). Post-menopausal women with node negative, ER+ cancers $3 \mathrm{~cm}$ or less were randomized on the Stockholm Tamoxifen Trial (STO-3) to either 2 or 5 years of Tamoxifen, or no systemic therapy. MammaPrint was performed on stored tumor samples from these patients. The breast cancer specific survival (BCSS) for the untreated patients with MammaPrint indices of $>0.355$ was $94 \%$ at 20 years, compared to $97 \%$ for those receiving 2 or more years of Tamoxifen. For those women with Low Risk MammaPrint indices not in this "ultra low" or Late Recurrence Low Risk (LRLR) range, endocrine therapy was highly beneficial and significantly improved survival. Pathology features such as grade and Ki67 were unable to identify these subsets.

Within the MammaPrint High Risk range, indices 0.00 to- 1.00 , endocrine therapy appears to be inadequate for substantially reducing the risk of recurrence, and additional therapy is needed. Data from the neoadjuvant I-SPY 2 Trial (Investigation of Serial Studies to Predict Your Therapeutic Response With Imaging And molecular Analysis 2) has identified differential response to chemotherapy and targeted therapies for cancers with MammaPrint High Risk indices at the lower portion of the range ("High 1 ") compared to those at the upper portion of the range ("High2") (36-38). Cancers 
with a MammaPrint High 2 index were highly likely to obtain pathologic complete remission(pCR) with the PARP inhibitor veliparib combined with carboplatin $(36,37)$, as well as to immunotherapy with the pembrolizumab combined with paclitaxel (38). MammaPrint High2 identified ER+ breast cancers likely to respond to pembrolizumab, a therapy with little activity in unselected $\mathrm{ER}+$ breast cancers.

\section{Conclusion}

The MINDACT trial provided the first reported prospective randomized data supporting the clinical utility of the MammaPrint 70 gene assay in early stage breast cancer and will provide a rich source of additional data in the years to come as further sub studies and additional follow-up are performed. Gene expression profiling with MammaPrint has the ability to identify the risk of early metastasis, the likelihood of long term disease specific survival without therapy, and the likelihood of response to targeted therapies. With such clinically important information derived from only 70 genes, the potential information which could be obtained from analysis of the full transcriptome may be extraordinary. Full transcriptome expression data can now be obtained from any breast cancer also undergoing MammaPrint testing, offering the opportunity to explore a virtually unlimited array of important questions in breast cancer. This valuable information is now being collected through a registry trial sponsored by Agendia known as FLEX (Full-genome Data Linked with Clinical Data to Evaluate New Gene Expression Profiles) in which full transcriptome data will be correlated with extensive clinical annotation (see Trivedi et al, in this issue). Breast cancer clinicians may eventually rely on access to the anatomy of the full genome for the practice of precision oncology, a process which began with the 70 gene MammaPrint assay.

Authors' Contributions: Conception and design: WA, LB, HK, GS and HT; Acquisition, analysis and interpretation of data: WA, LB and HK; Drafting the article: WA; Revising it critically for important intellectual content: WA, LB and HK; Approved final version of the manuscript: WA, LB, HK, GS and HT.

Conflict of Interest: Dr William Audeh: Chief Medical Officer, Agendia, Inc.; Dr Lisa Blumencranz, Senior Medical Science Liaison, Agendia, Inc.; Dr Heather Kling, Medical Science Liaison, Agendia, Inc. Other authors declare that they have no conflict of interest.

\section{References}

1. Bray F, Ferlay J, Soerjomataram I, Siegel RL, Torre LA, Jemal A. Global cancer statistics 2018: GLOBOCAN estimates of incidence and mortality worldwide for 36 cancers in 185 countries. CA Cancer J Clin. 2018;68(6):394-424.

2. van 't Veer LJ, Dai $H$, van de Vijver MJ, He YD, Hart AA, Mao M, et al. Gene expression profiling predicts clinical outcome of breast cancer. Nature. 2002;415(6871):530-6.

3. Cardoso F, van't Veer LJ, Bogaerts J, Slaets L, Viale G, Delaloge S, et al. 70-Gene Signature as an Aid to Treatment Decisions in Early-Stage Breast Cancer. N Engl J Med. 2016;375(8):717-29.

4. Hudis CA, Dickler M. Increasing Precision in Adjuvant Therapy for Breast Cancer. N Engl J Med. 2016;375(8):790-1.

5. Retèl VP, Joore MA, Knauer M, Linn SC, Hauptmann M, Harten WH. Cost-effectiveness of the 70 -gene signature versus St. Gallen guidelines and Adjuvant Online for early breast cancer. European Journal of Cancer. 2010;46(8):1382-91.

6. Krop I, Ismaila N, Stearns V. Use of Biomarkers to Guide Decisions on Adjuvant Systemic Therapy for Women With Early-Stage Invasive Breast Cancer: American Society of Clinical Oncology Clinical Practice Focused Update Guideline Summary. J Oncol Pract. 2017;13(11):763-6.

7. Giuliano AE, Edge SB, Hortobagyi GN. Eighth Edition of the AJCC Cancer Staging Manual: Breast Cancer. Ann Surg Oncol. 2018;25(7):83-5.

8. Curigliano G, Burstein HJ, E PW, Gnant M, Dubsky $\mathrm{P}$, Loibl S, et al. De-escalating and escalating treatments for early-stage breast cancer: the St. Gallen International Expert Consensus Conference on the Primary Therapy of Early Breast Cancer 2017. Ann Oncol. 2017;28(8):1700-12. 
9. Gradishar WJ, Anderson BO, Balassanian R, Blair SL, Burstein HJ, Cyr A, et al. NCCN Guidelines Insights: Breast Cancer, Version 1.2017. J Natl Compr Canc Netw. 2017;15(4):433-51.

10. Bogaerts J, Cardoso F, Buyse M, Braga S, Loi S, Harrison JA, et al. Gene signature evaluation as a prognostic tool: challenges in the design of the MINDACT trial. Nat Clin Pract Oncol. 2006;3(10):540-51.

11. van de Vijver MJ, He YD, van't Veer LJ, Dai $H$, Hart AA, Voskuil DW, et al. A gene-expression signature as a predictor of survival in breast cancer. N Engl J Med. 2002;347(25):1999-2009.

12. Buyse M, Loi S, van't Veer L, Viale G, Delorenzi M, Glas AM, et al. Validation and clinical utility of a 70-gene prognostic signature for women with node-negative breast cancer. J Natl Cancer Inst. 2006;98(17):1183-92.

13. Bueno-de-Mesquita JM, Linn SC, Keijzer R, Wesseling J, Nuyten DS, van Krimpen C, et al. Validation of 70-gene prognosis signature in nodenegative breast cancer. Breast Cancer Res Treat. 2009;117(3):483-95.

14. Tian S, Roepman P, van't Veer LJ, Bernards R, De Snoo F, Glas AM. Biological Functions of the Genes in the Mammaprint Breast Cancer Profile Reflect the Hallmarks of Cancer. 2010;5:BMI. S6184.

15. McGee SF, Lanigan F, Gilligan E, Groner B. Mammary gland biology and breast cancer. Conference on Common Molecular Mechanisms of Mammary Gland Development and Breast Cancer Progression. EMBO Rep. 2006;7(11):1084-8.

16. Pollack A, Test to Predict Breast Cancer Relapse is Approved [cited 2007 Feb 7]. New York Times. Available from: https://www.nytimes. com/2007/02/07/health/07breast.html.

17. Ravdin PM, Siminoff LA, Davis GJ, Mercer MB, Hewlett J, Gerson N, et al. Computer program to assist in making decisions about adjuvant therapy for women with early breast cancer. J Clin Oncol. 2001;19(4):980-91.

18. Duric VM, Stockler MR, Heritier S, Boyle F, Beith J, Sullivan A, et al. Patients' preferences for adjuvant chemotherapy in early breast cancer: what makes AC and CMF worthwhile now? Ann Oncol. 2005;16(11):1786-94.

19. Ravdin PM, Siminoff IA, Harvey JA. Survey of breast cancer patients concerning their knowledge and expectations of adjuvant therapy. J Clin Oncol. 1998;16(2):515-21.

20. Duric V, Stockler M. Patients' preferences for adjuvant chemotherapy in early breast cancer: a re- view of what makes it worthwhile. Lancet Oncol. 2001;2(11):691-7.

21. Zimmermann C, Baldo C, Molino A. Framing of outcome and probability of recurrence: breast cancer patients' choice of adjuvant chemotherapy (ACT) in hypothetical patient scenarios. Breast Cancer Res Treat. 2000;60(1):9-14.

22. Early Breast Cancer Trialists CG. Effects of chemotherapy and hormonal therapy for early breast cancer on recurrence and 15-year survival: an overview of the randomised trials. Lancet. 2005;365(9472):1687-717.

23. Early Breast Cancer Trialists' Collaborative G, Peto R, Davies C, Godwin J, Gray R, Pan HC, et al. Comparisons between different polychemotherapy regimens for early breast cancer: meta-analyses of long-term outcome among 100,000 women in 123 randomised trials. Lancet. 2012;379(9814):432-44.

24. Hudis CA, Barlow WE, Costantino JP, Gray RJ, Pritchard KI, Chapman JA, et al. Proposal for standardized definitions for efficacy end points in adjuvant breast cancer trials: the STEEP system. J Clin Oncol. 2007;25(15):2127-32.

25. Chen E, Tong KB, Malin JL. Cost-effectiveness of 70-gene MammaPrint signature in node-negative breast cancer. Am J Manag Care. 2010;16(12):e33342.

26. Cardoso F, Piccart-Gebhart MJ, Rutgers EJ, Litière $\mathrm{S}$, Veer LVt, Viale G, et al. Standard anthracyclinebased vs. docetaxel-capecitabine in early breast cancer: Results from the chemotherapy randomization (R-C) of EORTC 10041/ BIG 3-04 MINDACT phase III trial. J Clin Oncol. 2017;35(15 Suppl):516.

27. Kraemer HC, Blasey C, How many subjects?: statistical power analysis in research. 2 nd ed. Los Angeles: Sage Publicastions, Inc.; 2016.

28. Merkel D, Osborne CK. Use of steroid receptor assays in cancer management. Rev Endocrinol Rel Cancer. 1988;30:5-12.

29. Vogel CL, Cobleigh MA, Tripathy D, Gutheil JC, Harris LN, Fehrenbacher L, et al. Efficacy and safety of trastuzumab as a single agent in firstline treatment of HER2-overexpressing metastatic breast cancer. J Clin Oncol. 2002;20(3):719-26.

30. Fehrenbacher L, Cecchini RS, Geyer CE, Rastogi P, Costantino JP, Atkins JN, et al. NSABP B-47 (NRG oncology): Phase III randomized trial comparing adjuvant chemotherapy with adriamycin (A) and cyclophosphamide (C) $\rightarrow$ weekly paclitaxel (WP), or docetaxel (T) and $\mathrm{C}$ with or without a year of trastuzumab $(\mathrm{H})$ in women with nodepositive or high-risk node-negative invasive breast 
cancer (IBC) expressing HER2 staining intensity of IHC $1+$ or $2+$ with negative FISH (HER2-Low IBC) [abstract]. In: Proceedings of the 2017 San Antonio Breast Cancer Symposium; 2017 Dec 5-9; San Antonio, TX. Philadelphia (PA): AACR. Cancer Res. 2018;78(4 Suppl):Abstract nr GS1-02.

31. Knauer M, Mook S, Rutgers EJ, Bender RA, Hauptmann M, van de Vijver MJ, et al. The predictive value of the 70-gene signature for adjuvant chemotherapy in early breast cancer. Breast Cancer Res Treat. 2010;120(3):655-61.

32. Goetz MP, Gradishar WJ, Anderson BO, Abraham J, Aft R, Allison KH, et al. NCCN Guidelines Insights: Breast Cancer, Version 3.2018. J Natl Compr Canc Netw. 2019;17(2):118-26.

33. Sparano JA, Gray RJ, Makower DF, Pritchard KI, Albain KS, Hayes DF, et al. Adjuvant Chemotherapy Guided by a 21-Gene Expression Assay in Breast Cancer. N Engl J Med. 2018;379(2):111-21.

34. Dowsett M, Turner N. Estimating Risk of Recurrence for Early Breast Cancer: Integrating Clinical and Genomic Risk. J Clin Oncol. 2019;37(9):68992.
35. Esserman LJ, Yau C, Thompson CK, van 't Veer LJ, Borowsky AD, Hoadley KA, et al. Use of Molecular Tools to Identify Patients With Indolent Breast Cancers With Ultralow Risk Over 2 Decades. JAMA Oncol. 2017;3(11):1503-10.

36. Rugo HS, Olopade OI, DeMichele A, Yau C, van 't Veer LK, Buxton MB, et al. Adaptive Randomization of Veliparib-Carboplatin Treatment in Breast Cancer. N Engl J Med. 2016;375(1):23-34.

37. Wolf DM, Yau C, Sanil A, Glas A, Petricoin E, Wulfkuhle J, et al. DNA repair deficiency biomarkers and the 70-gene ultra-high risk signature as predictors of veliparib/carboplatin response in the I-SPY 2 breast cancer trial. NPJ Breast Cancer. 2017;3:31.

38. Yau C, Wolf D, Brown-Swigart L, Hirst G, Sanil A, Singhrao R, et al. Analysis of DNA repair deficiency biomarkers as predictors of response to the PD1 inhibitor pembrolizumab: Results from the neoadjuvant I-SPY 2 trial for stage II-III highrisk breast cancer [abstract]. In: Proceedings of the 2017 San Antonio Breast Cancer Symposium; 2017 Dec 5-9; San Antonio, TX. Philadelphia (PA): AACR. Cancer Res. 2018;78(4 Suppl):Abstract nr PD6-14. 